\title{
Tavşan, kobay ve sıçanda testisin şekli, konumu ve testis epididymis ilişkisi
}

\author{
Ahmet ÇAKIR ${ }^{1}$, Emine Ü. BOZKUR'T ${ }^{2}$, Ayhan DÜZLER ${ }^{3}$ \\ 'Ankara Üniversitesi. Veteriner Fakiiltesi. Anatomi Anabilim Dal, Ankara: ${ }^{2}$ Harran C̈niversitesi, Veteriner Fakuiltesi. Anatom! \\ Anabilion Dalı. Şanlıurfa; ${ }^{3}$ Ercjyes Üniversitesi. Veteriner Fakültesi. Anatomi Anabilim Dalı. Kayscri
}

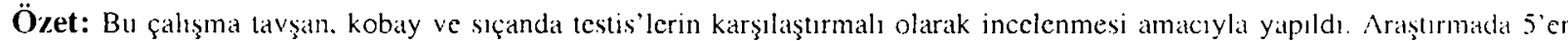
adel ergin tavşan (Beyaz. Yeni \%elanda), kobay (albino) ve sıçan (Sprague-Dawley) kullanildı. Her ü̧̧ türde de testis lerin iki arkat

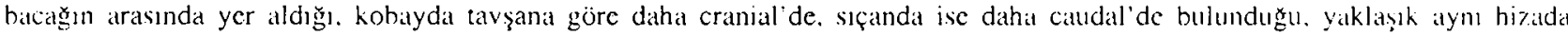

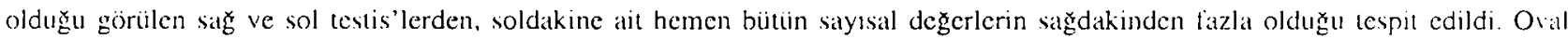
görüunimlü ıcstis'lerin, tavşanda yanlardan basık, kobayda dolgun, sıçanda isc yuvarlağa yakın olarak ş̧ckillcndiği saptandı. Corpus epididymidis'in tavşanda testis'in ventrolateral'inde. kobay ve sı̧̧anda isc dorsolateral'inde yer aldığ belirlendi. Tavşanda bursat testicularis butunamad.

Anahtar kelimeler: Anatomi, epididymis, kobay, sıçan, tavş̧an, testis

\section{Shape and location of testis in rabbit, guinea pig and rat and its relation with epididymis}

Summary: The aim of this study was to investigate the testes in rabbit, guineal pig and rat, comparatively. Five mature rabbit (White New Zcaland), guinca pig (albino) and rat (Sprague-Dawley) were used in this research. Testes were observed between hind limbs of these three species. They were located more cranially in guinea pig and more caudally in rat than those of rabbit. Left and right testis were situated nearly at the same level. It was concluded that, measurement values of the right testis were generally highel than those of left one. The testes were ovoid in form and compressed from side to side in rabbit, more swollen in guinca pig than thall of rabbil and they were nearly round in rat. The body of epididymis was seen ventrolaterally of the testis in rabbit and dorsolaterally in guinca pig and rat. The testicular bursa was not found in rabbit.

Key words: Anatomy, epididymis, guinea pig, rabbit, rat, testis

\section{Giriş}

Testis'ler cinscl olgunluğ crişmiş erkeklerde spermato\%oa üretimini sağlamakıan sorumlu olan organlardır $(4,8)$. Scrotum denilen deri kese içerisinde çift olarak bulunurlar $(2,6)$. Endokrin ve ekzokrin komponentleri kapsarlar. Hormonal faaliyctler vücut sıcaklığında yüriịchilir, ancak spermatozoa üretimi için birkaç derece düşïk ısıya ihtiyaç vardır. Bunun için de birçok hayvanda fetal dönemde cavum abdominis'te bulunan testis'ler doğumdan bir süre sonra scrotum'a göç cderler (3). Siçanda oval şekilli olan testis'ler doğun sonrası bir ay kadar daha karın boşlugunda kalır (13).

Testis'le ilgili olarak, tekede (1), boğalarda $(7,10)$, yaban lavşanında (11) ve develerde (12) yapılmış çalısmalar bulunnakıadır.

Son yillarda Türkiye'de özellikle insan hekimliğinin her alanında laboratuvar hayvanları üzerinde yapılan çalışmalarda artı̧̧ olduğu ve araştırmacıların anatomik kaynak arayışı içine girdikleri gözlennmektedir. Bu çalışmada tavşan, kobay ve sıçanda türün devamı açısından çok önemli olan testis'lerin anatomik özelliklerinin karşılaştırmalı olarak incelenmesi amaçlanmıştır.

\section{Materyal ve Metot}

Araştırmada, daha önce başka çalışmalarda degerlendirilmiş 5'er adet ergin tavşan (Beyaı Yeni Zelanda), kobay (albino) ve sıçan (Sprague-Dawley) kullanılmıştır. Dışarıdan scrotum'un yeri belirlendikten sonra discksiyonla testis'ler açığa çıkarılnış ve bulgular alınmıştır. Ağırlık ve hacin ölçümlerinde tunica vaginalis ve dışında kalan yapılar hariç tutulnış, funiculus spermaticus'un anulus inguinalis superficialis'e kadar olan bölümünü oluşturan diğer oluşumlar ise testis'le birlikte değgerlendirilmiştir. Ölçümlerde dijital kumpas, hassas terazi ve cam mezür kullanılmışur. Anatomik terimlerde Nomina Anatomica Veterinaria'dan (9) yararlanılmışır.

\section{Bulgular}

Tavşanda testis'lerin (Şckil la) arka iki bacak arasında, iki ayrı deri kese (scrotum) içinc ycrleştiği göriildui Scrotum kaldırıldığında testis'in extremitas caudata'sının caudolateral'e doğru kıvrıldığı, iki testis arasında penis'in (Şckil lb) uzandığı saptandı. Testis'lerin hemen hemen aynı hizada oldukları belirlendi. Tunica vaginalis (Şekil 1c) açıldığında cauda epididymidis'in (Şckil Id) caput 
epididynidis'ten (Şekil le) daha büyük olduğu, corpus epididymidis'in (Şekil 1f) ise tcstis'in ventrolateral'inde scyretliğ gözlendi. Caput epididymidis' in extremitas capitata'yı bir miğger gibi örttüği saptandı. Bursa testicularis tespit edilemedi. Tavşanda funiculus spermaticus’un (Şekil lg) kobay ve sıçana oranla daha uzun olcluğgu görüldü. Ductus deferens’in (Şekil $1 \mathrm{~h}$ ) corpus epididymidis'in dorsal'inde bulundugu saptand. Testis'in uzun ekseninin vücudun uzun eksenine paralel olmadığı, birà' çapraz pozisyonda (craniomedial -caudolateral) yerleşliğ ve testis'in yassı oval şekilli olduğu belirlendi. Her üç hayvan türüne ait testis ile ilgili ölçümler Tablo l'de gösterilmiştir.

Kobayda testis'lerin (Şekil 2a) arka iki bacak arasında, tavşan ve sıçan'a görc biraz. önde, penis'in (Şekil 2b) craniolateral'inde oldukça belirgin tek bir kese içine yerleştiğ görüldü. Testis'lerin extremitas caudata'sının (Şckil 2c) biraz dorsal'e doğru kalkık olduğu, iki testis'in aym hizada bulunduğu saptandı. Scrotum ve tunica vaginalis (Şckil 2d) açıldığında exuremitas capitata (Şckil 2c) çcvresinde büyük bir yağ doku kitlesinin (Şekil 2f) bulunduğu, caput epididymidis' in belirgin bir şekildc küçük oldugu, corpus epididymidis'in (Şekil 2g) ise testis'in dorsolateral inde yer aldığ 1 tespit edildi. Oval şckilli testis' in tavşanınkine göre daha dolgun olduğu göriildii. Corpus epididymidis ile testis arasında hursa testicularis'in bulunduğu ve ağız kısmının medial'e bakı̆̆ gö\% lendi. Diğer iki türdekinden daha büyik olarak şekillenen cauda epididymidis' in (\$ekil 2h) uç kısmunda, ducıus deferens (Şekil 21) başlamadan önce ductus epididymidis in kıvrımılarının çok belirgin olduğu, ductus deferens' in de testis'in dorsal'inde başlangıçta birkaç kıvnm gösterdikten sonra düzleşerek cranial'e doğru u\%andığ tespil edildi. Kobayda testis’lerin tavşan ve sıçana göre daha büyük oldugu saptandı.

Sıçanda testis'lerin (Şekil 3a) diğger iki türe oranla daha geride ancak birbirine yakın olarak bulunduğu ve preputium ile anus arasına yerleştiği gözlendi. (Preputium - anus arası mesafe ortalama $26 \mathrm{~mm}$ ). Tek bir kese halindeki scrotum'un caudal ucumun anus 'ii veneral'den orttüğü belirlendi. Scrotum üzerinde iki testis arasında ca-

Tablo 1. Tavşan. kobay ve sıçanda testis'e ait ortalama ölçümler. Table 1 . Average measurements of the testis in rabbit, guinea pig and rat.

\begin{tabular}{|c|c|c|c|c|c|c|c|}
\hline Materyal & $\begin{array}{l}\text { T.a } \\
\text { (gr) } \\
\end{array}$ & $\begin{array}{c}\text { T.h. } \\
(\mathrm{mm})\end{array}$ & $\begin{array}{c}\text { T.h. } \\
\left(\mathrm{cm}^{3}\right)\end{array}$ & $\begin{array}{c}\text { T.ç. } \\
(\mathrm{mm})\end{array}$ & $\begin{array}{l}\text { C.c.g. } \\
(\mathrm{mm})\end{array}$ & $\begin{array}{c}\text { D.d.d.ç. } \\
(\mathrm{mm})\end{array}$ & $\begin{array}{c}\text { C.c.c.d.u } \\
(\mathrm{mm})\end{array}$ \\
\hline Tavş̧all-săğ & 7.2 & 21.1 & 5.5 & 10.2 & 2.2 & 1.7 & 29.3 \\
\hline Tavşan-sol & 7.8 & 20.5 & 6 & 10.8 & 2.3 & 1.8 & 30.3 \\
\hline Kobay-satg & 7.9 & 23.6 & 9.5 & 13.4 & 2.7 & 2.4 & 33.8 \\
\hline Kobay-sol & 8.6 & 23.7 & 10.5 & 13.8 & 1.9 & 1.8 & 34.2 \\
\hline Siçan-Sią̆ & 4.6 & 18.0 & 4 & 8.9 & 2.5 & 1.7 & 30.9 \\
\hline Siçan-sol & 4.8 & 19.3 & 4 & 9.3 & 2.4 & 1.6 & $3(1) .8$ \\
\hline
\end{tabular}

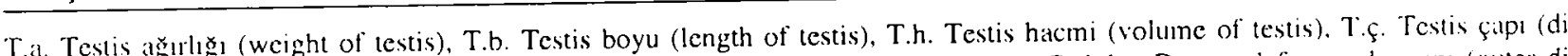
ameter of testis). C.c.g. Corpus epididyınidis genişliğ (width of corpus epididymis). D.d.d.ę. Ductus deferens dı̧ çapı (outer di a

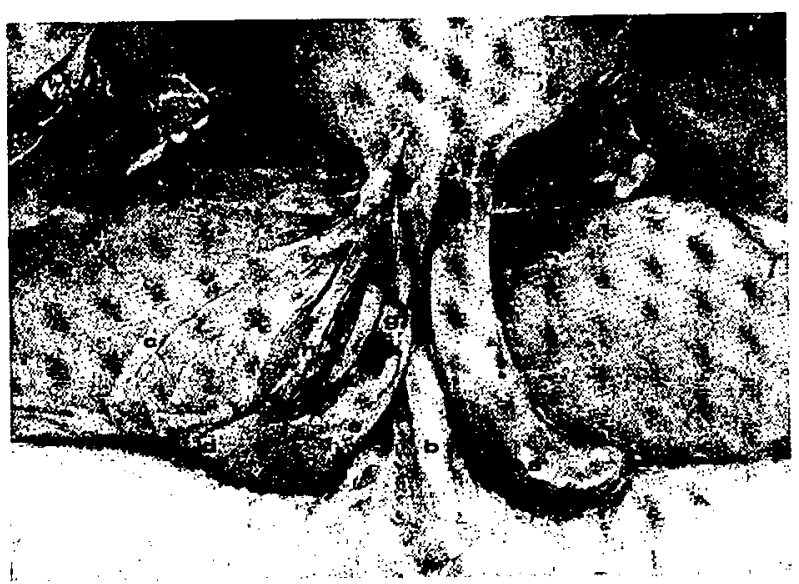

Sekil 1. Tat'sanda testis'lerin ventral den gorüniişii

Figure 1. Ventral view of the testes in rabbit.

a. Testis. b. Penis, c. Tunica vaginalis. d. Cauda epididymidis. c. Capul epididymidis. f. Corpus epididymidis. g. Funiculus spermaticus. h. Ducuss deferens.

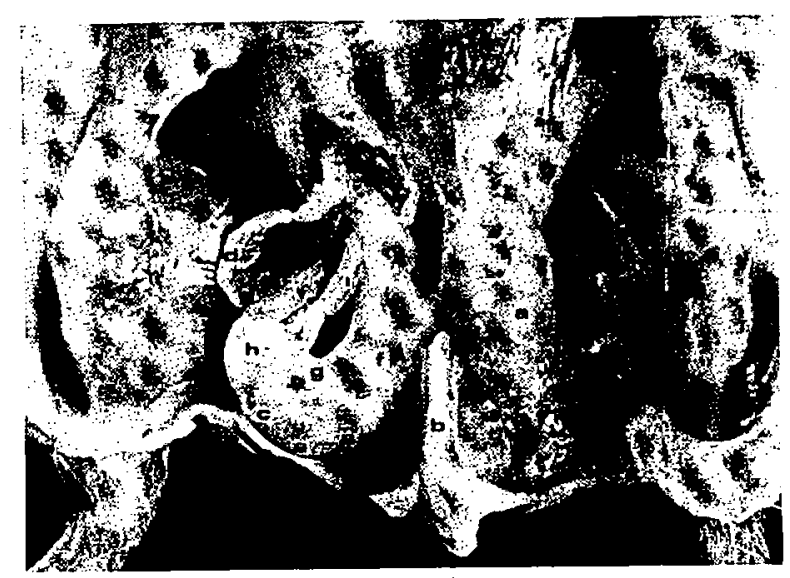

Şekil 2. Kobayda testis' Ierin ventral' den goriminişii. Figure 2. Ventral view of the testes in guineat pig

a. Testis, b. Penis, c. Extremitas caudata, d. 'Tunica vaginalis. c. Extremitas capitatit. $t$. Yag doku (adipose tissue). Corpus epididymidis, h. Cauda epididymidis. 3. Ducuus deferens. 


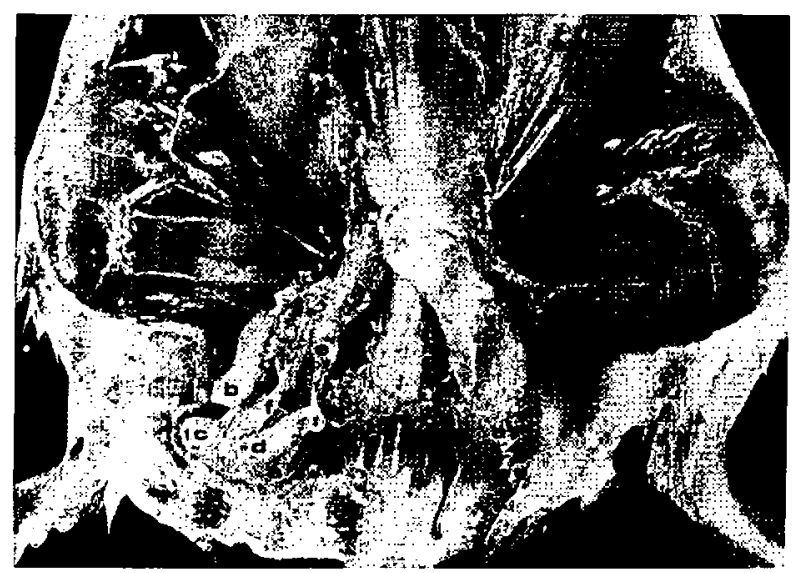

Şekıl 3. Sıçandat tessus'|erin ventral'den görïinuşüi.

Figure 3. Ventail view of the testes in rat.

a. Testis b. Tunicat vaginalis, c. Cauda epididymidis, d. Corpus epididymidis, c. Caput epididymidis, f. Ductus deferens.

udal'de daha belirgin bir oluğun yer aldığı tespit edildi. Scrotum ve tunica vaginalis (Sckil 3b) açıldığında tcstis 'in küı oval şckilli (yuvarlağa yakın) olduğgu, cauda epididymidis (Sckil 3c) ilc birlikte extremitas caudata'nın medial'e doğru biraz kıvrıldığ (Sckil 3d) ise testis'in dorsolateral'inde bulunduğu saptand. Caput epididymidis' in (Şekil 3e) tavşanda olduğu gibi testis in extremitas capitata’sını bir miğfer tarzında örttiğü ve diğer iki türe görre daha büyük şekillendiği gözlendi. Bu oluşunun çevresinde az miktarda yağ dokumun yer aldı ğ belirlendi. Ductus deferens'in (Şekil 3f) corpus epididymictis'in dorsal'inde olduğu saptand1. Sif̧anda bursa testicularis'in bulunduğu tespit edildi.

\section{Tartışma ve Sonuç}

Scrotum'un, dolaysstyla testis`lerin ruminantia'da ahdomen'in gerisinde, kedi ve domuzda perineal bölgede, köpek ve atla ise bunlara göre intermedier bölgede yer aldığ helirtilmiştir (3). McLaughlin (6) tavşanda testis'lerin pelvis in ventral'inde. penis'in ise cranial'inde bulundığ mada lestis'lerin lavşanda belittilenden farklı olarak penis" in her iki yanunda iki ayrı kese içine yerleştiği, kobayda tavşana göre daha cranial'de, sıçanda ise daha caudal' de bulunduğu saptandı.

Hemen hemen bütün türlerde sol testis'in sağ testis'ten büyük olduğu (3), boğada testis"lerin boyutlarının değgişcbileceği, birinin diğcrine göre daha dorsal'de yer aldığı belirtilmiştir (8). Yapılan ölçümlerde her üç hayvanda da sol testis'c ait hemen bütün değerlerin sağdakinden fazla olduğu tespil edildi. Her iki testis'in ü̧̧ hayvan türunde de yaklaşık olarak aym hizada olduğu göriilclii.
Bazı rodent, böcekyiyen ve yarasalarda testis" lerin uireme döneminde scrotum'a inip sonra tekrar karın boşluğuna döndükleri belirlenmiştir (3). Yahan tavşanı üze rinde yapılan bir çalışmada testis lerin temmuz eylül ay ları arasında scrotum'da olduğu, ekim ve kasım aylarında bazı tavşanlarda canalis inguinalis`e doğgru çckildikleri saptanmışur (11). Siçanda ise diğer rodenl lerde olduğ gibi testis'lerin inaktif periyoula karm boşluğuna çe kilebileceği ifade edilmiştir (5). Araştırnada kullanılàn hayvanların hepsinde testis"lerin scrotum içinde yer aldığ tespit edildi.

Testis' lerin oval şckilli olduğu $(4,8,13)$, atta aynı zamanda yanlardan basık olarak bulunduğu belirtilmiştir (4). Calışmada her üç türde de oval olduğu göriilen lestis'lerin, lavşanda yanlardan basık. kobayda dolgun. s1çanda isc yuvarlağa yakın olarak şekillendiğ saptandı.

Testis ile corpus epididymidis arasinda bursa testicularis'in bulunduğu $(2,3)$ ve ağżmın tïrlere göre lateral'e veya caudal'e açıldığ çalışmada tavşanda bursa testicularis görülemedi. Kobay ve sıçanda varlığı saptandı ve ağıı kısmının medial c baktığ belirlendi.

Sonuç olarak, tavşanda testis"lerin kobaya oranla daha caudal'de. sıçana göre ise biraz cranial de yer aldığ uiç tuirde de testis'lcrin yaklaşık aynı hi\%ada olduğ $u$, kobayda testis"lerin, sıçanda ise caput epididymidis 'in büyük olarak şekillendiği, corpus epididymidis'in kobay ve sıçanda testis'in dorsolateral'inde, tavşanda ise ventrolateral'inde bulunduğu tespit edildi. Tavşanda bursa testicularis bulunamad.

\section{Kaynaklar}

1. Baishya G, Ahmed S, Bthattacharya M (1986): A correlative study on biometry and histomorphometry of inalegonad and thyroid gland $10-90$ days; in Arsam Gout (Capra hircus). Indian Vet J, 63, 928-932.

2. Dursun $\mathbf{N}$ (1994): Veteriner Anatomi II Medisan Yayınevi, Ankara.

3. Dyce KM, Sack WO, Wensing C.JG (1987): Texthork of Veterinary Anatomy: WB Saunders Company. Philidelphia.

4. Getty R (1975): The Anatomy of the Domesric: Anmuls. Fifth Edition. WB Saunders Company, Philadelphia.

5. Green EC (1963): Anutomy of the Rat. Hatiner Publishing Company. Ncw York.

6. McLaughlin AC, Chiasson BR (1979): Luboratory Anatomy of the Rubhit. Second Edition. Wm C Brown Company Publishers, lowa.

7. Narasimha RAV, Venkatramiah P (1993): Testicular biometry in growing Murrah Bulls. Indian VCl J. 70. 974 975.

8. Nickel R, Schummer A, Seiferle E (1981): The Anatom! of the Domestic Animals. Vol 3. First Edition. Verlag Patul Parcy, Bcrlin. 
9. Nomina Anatomica Veterinaria (1994): Prepared by the International Committee on Veterinan Gross Anatomical Nomentature and Authorized by the Eighteenth General Assembly of the World Association of Veterinany Anatomist. Fourth Edition. Vew York.

10. Pal C, Bharadwaj MML (1983): Morphology and biometry of the testis in Indian buffalo (Bubalus bubalis). Philipp J Vct Mcd. 22, 7-12.

11. Simeunovic B, Strbenc M, Bavdek SV (2000): Position and histological structure of the testes in the brown hare
(Lepus europaeus) during seasonal regression and recrudescence. Anat Histol Embryol. 29, 73-82.

12. Tingari MD, Ramos AS, Gaili ES, Rahma BA, Saad AH (1984): Morphology of the testis of the one-humped camel in relation to reproductive activit: J Anat. 139, 133-143

Yazışma adresi:

Doş: Dr. Ahmet Çakır

Ankara Universitesi Veteriner Fakültest

Anatomi Anahilim Dals

O6110, Ankara 